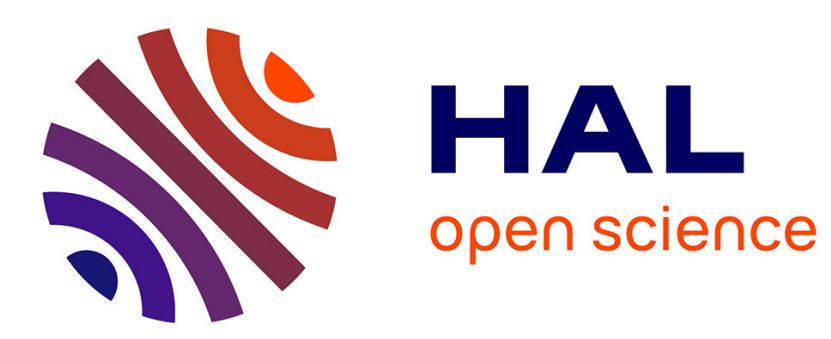

\title{
Genetics of cystic fibrosis: CFTR mutation classifications toward genotype-based CF therapies.
}

Pascale Fanen, Adeline Wohlhuter-Haddad, Alexandre Hinzpeter

\section{To cite this version:}

Pascale Fanen, Adeline Wohlhuter-Haddad, Alexandre Hinzpeter. Genetics of cystic fibrosis: CFTR mutation classifications toward genotype-based CF therapies.: Genetics of CF. International Journal of Biochemistry and Cell Biology, 2014, 52, pp.94-102. 10.1016/j.biocel.2014.02.023 . inserm-01059046

\section{HAL Id: inserm-01059046 https://www.hal.inserm.fr/inserm-01059046}

Submitted on 29 Aug 2014

HAL is a multi-disciplinary open access archive for the deposit and dissemination of scientific research documents, whether they are published or not. The documents may come from teaching and research institutions in France or abroad, or from public or private research centers.
L'archive ouverte pluridisciplinaire HAL, est destinée au dépôt et à la diffusion de documents scientifiques de niveau recherche, publiés ou non, émanant des établissements d'enseignement et de recherche français ou étrangers, des laboratoires publics ou privés. 


\title{
Genetics of Cystic Fibrosis:
}

\section{CFTR Mutation Classifications towards Genotype-Based CF Therapies}

Pascale Fanen $^{1,2,3^{*}}$, Adeline Wohlhuter-Haddad ${ }^{2,4}$, Alexandre Hinzpeter $^{1,2}$

${ }^{1}$. Université Paris-Est, UMR_S955, UPEC, F-94000, Créteil, France; ${ }^{2}$. Inserm, U955, Equipe 11, F-94000, Créteil, France; ${ }^{3}$. AP-HP, Hôpital H. Mondor - A. Chenevier, Département de

Génétique, F-94000, Créteil, France; ${ }^{4}$. McGill University, Faculty of Medicine, Department of Physiology, Montréal, QC, Canada.

*Corresponding author: Pascale Fanen, MD, PhD, INSERM U955, Equipe 11

Hôpital Henri Mondor

Créteil, F-94010, France

Tel: +33149812854/+33149814822

Fax: +33148993345

E-mail: pascale.fanen@inserm.fr

Running title: Genetics of CF

Keywords: CFTR, mutation, CFTR-RD, therapy, Variants of Unknown significance

\begin{abstract}
Abbreviations: CBAVD, congenital bilateral absence of the vas deferens; $\mathrm{CF}$, cystic fibrosis; CFTR, Cystic Fibrosis Transmembrane conductance Regulator; CFTR-RD, CFTR related disease; COPD, chronic obstructive pulmonary disease; EJC, exon junction complex; NBD, nucleotide-binding domains; NGS, next generation sequencing; NPD, nasal potential difference measurements; NMD, Nonsense-Mediated mRNA Decay; PABP, Poly(A)-binding protein; PI, pancreatic insufficiency; PTC, premature termination codon; PS, pancreatic sufficiency; VUs, Variants of Unknown significance.
\end{abstract}




\begin{abstract}
Cystic fibrosis (CF) is an autosomal recessive disease caused by mutations in the cystic fibrosis transmembrane conductance regulator (CFTR) gene, which encodes an epithelial anion channel. Since the identification of the disease in 1938 and up until 2012, CF patients have been treated exclusively with medications aimed at bettering their respiratory, digestive, inflammatory and infectious symptoms. The identification of the CFTR gene in 1989 gave hopes of rapidly finding a cure for the disease, for which over 1,950 mutations have been identified. Since 2012, recent approaches have enabled the identification of small molecules targeting either the CFTR protein directly or its key processing steps, giving rise to novel promising therapeutic tools.

This review presents the current CFTR mutation classifications according to their clinical consequences and to their effect on the structure and function of the CFTR channel. How these classifications are essential in the establishment of mutation-targeted therapeutic strategies is then discussed. The future of CFTR-targeted treatment lies in combinatory therapies that will enable $\mathrm{CF}$ patients to receive a customized treatment.
\end{abstract}




\section{Introduction}

Mutations disrupting the function of the Cystic Fibrosis Transmembrane conductance Regulator (CFTR) gene, also referred to as $A B C C 7$, cause Cystic Fibrosis (CF), a severe autosomal recessive disease. $\mathrm{CF}$ affects 1 in 2,500 to 4,500 newborns among the Caucasian population. All other ethnic groups are affected to a lesser extent. In 1989, Francis Collins, Lap-Chee Tsui and John R. Riordan (Kerem et al., 1989) identified the CFTR gene and its most frequent mutation, F508del, as being the cause of CF. The CFTR gene is located on the long arm of chromosome 7 (7q31.2) and its structure comprises 27 exons, spanning over 190kb (Fig. 1A). After splicing of the large introns, the CFTR mRNA is $6.5 \mathrm{~kb}$ and all of its exons are necessary to produce a functional CFTR protein.

Since 1990, the laboratories dedicated to CF genetic diagnosis and research worldwide have been grouped into the international Cystic Fibrosis Genetic Analysis Consortium (CFGAC). This consortium identifies and describes mutations of the CFTR gene. Inputs are then deposited on the CF mutation database "CFTR1" (http://www.genet.sickkids.on.ca/cftr/) for public access. Since the discovery of the CFTR gene, extensive sequencing enabled the identification of over 1,950 different mutations. Recently, a second CF mutation database "CFTR2" for "Clinical and Functional Translation of CFTR", has been created (http://www.cftr2.org/). It gathers up-to-date information about newly discovered CFTR gene mutations, classifies them as disease-causing, neutral or mutation of varying clinical consequences, and provides clinical information about specific mutation combinations.

To better our understanding of CF pathogenesis, as well as to facilitate diagnosis and choice of treatment, two classification systems for CFTR mutations have been created, one according to clinical consequences, the other according to CFTR structure-function assessment. Currently available CF therapies mainly treat the symptoms of the disease, and have increased the mean life expectancy of CF patients from 5 years in the 1970s to about 35 to 40 years of age today. To address the underlying cause of the disease, recent approaches have enabled the identification of small molecules targeting either the CFTR protein directly, or its key processing steps. These novel therapeutic tools correct specific defects responsible for CFTR protein loss-of-function and need to be adapted to each patient's genotype. This highlights the importance of thoroughly characterizing each patient at the molecular level. 
This review first presents the clinical spectrum of Cystic Fibrosis, as well as the two existing classification systems for CFTR mutations. How CFTR mutations are assigned to CF patients is then described. The use of this essential information in the development of genotype-based therapeutic strategies is then discussed.

\section{Classification of CFTR mutations}

\subsection{The clinical spectrum of $C F$}

The first description of the disease was done in 1938 by Dorothy Hansine Andersen, describing an abnormal pancreas, which presented with cysts and fibrosis (Andersen, 1938). The clinical spectrum of $\mathrm{CF}$ has since greatly expanded, giving rise to diagnoses of classic and nonclassic CF presented in Figure 2 (De Boeck et al., 2006, Farrell et al., 2008).

Although classic CF most often presents with a severe multi-organ phenotype, and non-classic CF with milder single-organ phenotypes, this is not always the case. Whether the phenotype is "severe/mild" or "multi/single-organ" is not intrinsically linked to the classic or non-classic CF diagnosis. These are subjective terms, which should be used for descriptive purposes exclusively.

The great phenotypic variability of CF has been shown to implicate not only the type of CFTR mutations, but also other genetic factors such as modifier genes, (see chapter from Guillot and Corvol in this special issue) and environmental factors (lifestyle, treatment) (De Boeck et al., 2006). In the diagnostic algorithms proposed by the European Consensus Group, patients with one or more phenotypic characteristic suggestive of $\mathrm{CF}$ are classified according to their pilocarpine sweat test result (chloride $>60 \mathrm{mmol} / \mathrm{L}, 30-60 \mathrm{mmol} / \mathrm{L}$ or $<30 \mathrm{mmol} / \mathrm{L}$ ) (Castellani et al., 2008). To date, no test has proven to be as practical or reliable as the sweat test for clinical diagnostic purposes.

Classic CF - The majority of CF patients suffer from classic CF, yet their organs are affected to varying degrees. Patients are diagnosed with classic $\mathrm{CF}$ if they have one or more phenotypic characteristic and a sweat chloride concentration of $>60 \mathrm{mmol} / \mathrm{L}$ (Fig. 2). They may have exocrine pancreatic insufficiency (PI) or pancreatic sufficiency (PS). The disease can have a severe course with rapid progression of symptoms or a milder course with very little deterioration over time. Classic CF patient mortality is mainly due to progressive respiratory disease. From a genetic standpoint, classical CF is characterized by one established CF-causing mutation on each CFTR allele.

Non-classic CF - Non-classic CF describes individuals with at least one CF phenotypic 
characteristic and a normal $(<30 \mathrm{mmol} / \mathrm{L})$ or borderline $(30-60 \mathrm{mmol} / \mathrm{L})$ sweat chloride level, in whom detection of one mutation on each CFTR allele, or direct quantification of CFTR dysfunction by NPD measurement has been confirmed (Boyle, 2003, Rosenstein, 2003) (Fig. 2). Non-classic CF patients have either multi- or single-organ involvement. Most of them have exocrine PS and milder lung disease. As described by the CF Diagnostic Working Group, "some patients with single-organ involvement resulting from CFTR dysfunction may be more appropriately given an alternative diagnostic label, as recommended in the World Health Organization diagnostic list" (WHO, 2000). These alternative diagnoses for non-classic CF, or CFTR-related disorders, include isolated obstructive azoospermia, chronic pancreatitis, disseminated bronchiectasis, allergic bronchopulmonary aspergillosis, diffuse panbronchiolitis and sclerosing cholangitis, and all have at least one identified associated CFTR mutation, some of which are discussed hereafter.

\subsection{Classification of CFTR mutations based on clinical consequences}

In 2007, the Consensus Conference, organized by the European Cystic Fibrosis Society with the partnership of the European Society of Human Genetics and the EuroGentest Network of Excellence, allowed for the establishment of a classification of CFTR mutations, into the following four groups, according to their clinical consequences (Table 1). Group A comprises mutations that cause classic CF (CF-causing), Group B includes mutations that cause non-classic CF (associated to CFTR-related disorders), Group C comprises mutations with no known clinical consequences, and Group D consists of mutations with unknown or uncertain clinical relevance (also referred to as VUs) (Castellani et al., 2008).

Only a few of the hundreds of CFTR mutations identified to date have been shown to result in classic CF. Within a given ethnic group, specific mutations may be more frequent than others. Most CF patients are Caucasian, however, other ethnicities are also affected, and p.Phe508del remains the most frequent classic CF mutant allele, accounting for approximately $70 \%$ of worldwide chromosomes (Lucotte et al., 1995). As more and more patients present with nonclassic phenotypes, DNA screens for common CFTR mutations have become essential for CF diagnosis. To that effect, the American College of Medical Genetics (ACMG) has recommended a core panel of 23 mutations to assess CF risks in diagnosis, carrier screening and sequencing analyses (Table 2). These 23 mutations fall into Group A, as classic CF-causing mutations, with the exception of p.Arg117His, which is discussed later in a paragraph dedicated to complex 
alleles. This core panel may be complemented with the screening of additional ethnic-specific mutations found in European and/or other selected population. Some mutations may be associated with different clinical presentations. Groups A and B are therefore not mutually exclusive. For example, the p.Asp1152His mutation may result in isolated congenital bilateral absence of the vas deferens (CBAVD), or in a fully expressed CF lung disease with pancreatic sufficiency. Non-classic CF disorders have a pathophysiology that may be attributed to CFTR defect, as these patients are characterized by a prevalence of CFTR mutation higher than that expected in the general population.

Group D mutations have unknown or uncertain clinical relevance to CF phenotypic characteristics. It is often difficult to assess the association between CFTR mutations and clinical entities that, in some cases, may have other non-CFTR related aetiologies. Other factors, such as the environment, modifier genes and the progression of the disease with age, can also affect the clinical heterogeneity of patients carrying these large spectrum mutations.

It is important to note that CFTR genotype is not a useful predictor of the severity of lung disease in the individual patient, and should not be used as an indicator of prognosis (Castellani et al., 2008).

\subsection{Classification of CFTR mutations based on CFTR structure-function}

Five major classes of CFTR mutations were created according to their consequences on CFTR function (Welsh and Smith, 1993): mutations interfering with protein synthesis, mutations affecting protein maturation, mutations altering channel regulation, mutations affecting chloride conductance, and mutations reducing the level of normally functioning CFTR at the apical membrane (Welsh and Smith, 1993) (Fig. 3). A sixth class consisting of mutations decreasing the stability of CFTR present at the plasma membrane or affecting the regulation of other channels was proposed, but has been poorly investigated (Haardt et al., 1999). These class VI mutations are now combined with class $\mathrm{V}$, as mutations leading to a reduced amount of functional CFTR protein (Welsh et al., 2001).

\section{Class I: mutations preventing the production of a full-length CFTR protein}

Class I mutations result in the total or partial lack of production of a functional CFTR protein (Fig. 3). Such mutations may arise either due to (1) a nucleotide substitution introducing an inframe premature termination codon (PTC) -UAA, UAG or UGA-, (2) frame-shifting insertions or 
deletions, (3) mutations at the invariant dinucleotide splice junctions, or introduction of a PTC, resulting in complete skipping of an out of frame exon (Fig. 1B), (4) a complete or partial deletion of the CFTR gene or (5) a rearrangement in the gene altering the exon sequence. Mutations that generate PTCs can reduce the steady-state level of mRNA via nonsense-mediated decay (NMD), which degrades the abnormal PTC-containing mRNA (Maquat, 1995). Such a phenomena has been shown with p.Gly542*, p.Arg553*, p.Trp1282*, and one frameshift mutation (p.Phe316LeufsX12), but a notable exception is p.Arg1162*, which produces a normal CFTR mRNA level as compared to wild-type CFTR (Will et al., 1995).

The p.Gly542* mutation is the most frequent worldwide mutation of its class, affecting at least one allele of up to $4 \%$ of CF patients. Other mutations reach a higher frequency in specific populations, due to a founder effect; for example $48 \%$ of all alleles in Ashkenazi Jews in Israel carry the p.Trp $1282 *$ mutation and $24 \%$ carry the p.Tyr $122 *$ mutation in the French Reunion Island (Dugueperoux et al., 2004, Shoshani et al., 1992).

\section{Class II: mutations altering the cellular processing of the protein}

Class II mutations are associated with defective processing due to misfolding of the protein (Fig. 3) and may be found within any domain of the CFTR protein. The misfolded protein is retained in the Endoplasmic Reticulum (ER), retro-translocated to the cytoplasm, where it is degraded by the ubiquitin/proteasome pathway (Jensen et al., 1995, Ward et al., 1995). CFTR maturation through the cellular compartments can be assayed by western blot. The coreglycosylated CFTR (band B) migrates at a lower molecular weight than the fully-glycosylated CFTR protein (band C). Depending on the mutation, one may observe either a partial reduction (p.Leu206Trp, third transmembrane segment) or a complete absence (p.Arg1066Cys, fourth intracellular loop or p.Phe508del, NBD1) of mature CFTR. The most frequent CF mutation, p.Phe508del, has been well studied. It has been shown that p.Phe508del leads to energetic and kinetic instability of the NBD1 domain due to improper local folding (Protasevich et al., 2010). Additionally, CFTR domain assembly is altered, namely the interaction between NBD1 and the fourth cytoplasmic loop (CL4) (Cui et al., 2007, He et al., 2010). Correction of both NBD1 energetics and interface instability is therefore required to restore p.Phe508del processing (Mendoza et al., 2012, Rabeh et al., 2012). Initially, mutations belonging to this class were thought to cause severe CF similarly to p.Phe508del or p.Arg1066Cys, but p.Leu206Trp was shown to be associated with variable phenotype (Clain et al., 2005a). 


\section{Class III: mutations disturbing the regulation of the Cl channel}

These mutations are frequently located in the ATP binding domains (NBD1 and NBD2) and are referred to as gating mutations (Fig. 3). They are missense mutations producing a protein efficiently inserted in the membrane at normal levels, but resistant to activation by protein kinase A. The main example is p.Gly551Asp, which abolishes ATP-dependent gating, resulting in an open probability that is $\sim 100$-fold lower than that of the wild type channel (Anderson and Welsh, 1992). Other class III mutations such as the frequent mutations p.Arg560Thr or p.Gly970Arg are listed in Figure 3 (Seibert et al., 1996). Noteworthy, this latter mutation is located in the third cytoplasmic loop (CL3) of the protein, highlighting the fact that knowing the localization of the amino acid substitution is insufficient to predict the resulting CFTR structural and functional anomalies.

\section{Class IV: mutations altering the conduction of the Cl channel}

These mutations are mostly located within membrane spanning domains implicated in the constitution of the channel pore (Fig. 3). The missense mutations located in these regions produce a protein efficiently inserted in the membrane, which retains a cAMP-dependent $\mathrm{Cl}^{-}$channel activity, but with a reduced channel conductance. Alleles in this class are typically associated with a milder form of the disease. The p.Arg117His mutation is the best-characterized class IV mutation. While this mutant is correctly processed and generates cyclic AMP-regulated apical $\mathrm{Cl}^{-}$ currents, patch-clamp analysis demonstrated a reduction in both channel open probability and conductance (Reddy and Quinton, 2001, Sheppard et al., 1993).

Recently, a subclass IVb (b stand for bicarbonate) has been proposed for mutations affecting CFTR bicarbonate conductance specifically, without reducing its chloride conductance (Schneider et al., 2011). As of today, only a few of such mutants have been identified, namely p.Arg75Gln and p.Ile148Thr (Choi et al., 2001, Schneider et al., 2011). While p.Ile148Thr appears not to be deleterious (Claustres et al., 2004), p.Arg75Gln was found in some studies at a higher frequency in cohorts of patients presenting CFTR-RD (Divac et al., 2004, Schneider et al., 2011). Other mutations affecting the CFTR bicarbonate conductance are bound to be identified, probably in patients presenting with a CFTR-RD.

\section{Class V: mutations reducing the amount of functional CFTR protein}

Most of the class V mutations reduce the total amount of CFTR protein by affecting premRNA splicing (Fig. 3). These splice site mutations can induce complete or partial exclusion of 
an exon. In the latter case, production of normal mRNA is maintained, but in lower quantity. When the skipped exon is in phase (Fig. 1A), the transcript will produce an incomplete nonfunctional channel. On the other hand, when the skipped exon is out of phase, a PTC will rapidly appear and the transcript will be degraded by the NMD pathway. Classically, splicing mutations are located in the introns close to the splice sites, where they alter key splicing signals implicated in proper exon recognition (acceptor site, donor site, branch point or polypyrimidine tract). Yet, a growing number of them are being identified within exons. These exonic nucleotide substitutions can induce exon skipping by either disrupting Exonic Splicing Enhancer (ESE) or creating Exon Splicing Silencer (ESS) motifs (Pagani and Baralle, 2004). Since direct RNA analysis is not routinely performed, the number of mutations causing splicing defects is underestimated.

The most frequent and well-studied is the skipping of exon 10 (formerly named exon 9). The presence or absence of this exon is correlated with a polymorphism within the polypyrimidine tract located upstream of the acceptor splice site (Poly-T tract). While a Poly-T tract of 7T or 9T ensures up to $90 \%$ of normal splicing, the shorter 5T Poly-T tract allows no more than 10 to $40 \%$ of normal mRNA to be produced (Chu et al., 1993).

\section{Complex alleles and multi-class mutations}

Some CFTR alleles have the particularity of harbouring two distinct mutations. They are referred to as complex alleles. The two mutations can induce additive defects, leading to a more severe phenotype than would each mutation separately (Clain et al., 2001, Clain et al., 2005b). This can be illustrated by the class IV R117H mutation (c.350G >A, p.Arg117His) whose severity is modulated in cis by the 5/7/9T polypyrimidine tract (c.1210-12T(5_9)in intron 9. While the $\mathrm{R} 117 \mathrm{H}-\mathrm{T} 7$ genotype is associated with milder forms of CF such as CBAVD, and most of the time even absence of symptoms, the R117H-T5 can be identified in patients having elevated sweat chloride and clinical cystic fibrosis, which in some cases is severe (Thauvin-Robinet et al., 2009).

Finally, some point mutations have been shown to alter multiple distinct processes, such as splicing and routing or maturation and channel conductance. This is for example the case of the

p.Phe508del mutation, which presents, in addition to a processing defect, a gating defect characterized by a lower open probability of the channel and a reduced stability of the protein at the cell surface due to an increased recycling rate (Dalemans et al., 1991).

\subsection{Assigning CFTR mutations to CF patients: a methodological perspective}


As of today, only a minority of the 1950 known mutations have been analyzed at the protein level (Ferec and Cutting, 2012) and even fewer at the mRNA level. Studying the DNA/RNA obtained from clinically characterized CF patients has led to the identification of their mutations. With the upcoming introduction of NGS, the number of identified VUs will be greatly increased (Abou Tayoun et al., 2013, Trujillano et al., 2013). The labelling of these VUs, as diseasecausing or as neutral (Group C) mutations, will be challenging. Presented hereafter are some guidelines and available tools enabling such a distinction.

One such tool is the CFTR2 database, initiated to establish disease liability of individual CFTR mutations, as well as to determine the relationships between specific mutations and symptoms of CF. This project (http:/www.cftr2.org/) has compiled clinical data from nearly 40,000 CF patients and relates the clinical phenotype (pancreatic status, lung function, pulmonary infection) to the electrophysiological phenotype (sweat chloride and ion transport in epithelial cells transfected with the corresponding mutated CFTR). Among the 1221 mutations reported so far on the CFTR2 database, 190 mutations have already been characterized. For the remaining mutations, indirect evidence of pathogenic potential may be derived from the following (adapted from (Castellani et al., 2008)):

1) in vivo assessment of abnormal CFTR-mediated chloride transport, as assessed by sweat test or NPD and ex vivo assessment by short circuit measurements using Ussing chambers (nasal brushing or rectal biopsy),

2) molecular assessment of sequence variations

CFTR mutations with high confidence for pathogenic potential:

- change in an amino acid residue predicted to affect CFTR synthesis and/or function

- premature termination signal (insertion, deletion or nonsense mutation)

- alteration of the invariant dinucleotide of the donor or acceptor splice sites.

CFTR mutations with lower confidence for pathogenic potential:

- sequence variation detected in several unrelated individuals with CF

- change in an amino acid residue absent in at least 100 non-CF chromosomes of healthy carriers from the same ethnic group

- change in a highly evolutionarily conserved amino acid residue

- sequence variation creating/unmasking a cryptic splice site

- exonic or intronic sequence variation modifying splicing. 
When the observed criteria point to a mutation with lower confidence, additional tools and approaches are necessary to confirm or invalidate its pathogenic potential. The first approach is phenotype-driven, based on the analysis of well-phenotyped subjects with classic CF, thus helping to interpret the clinically relevant mutations (Sosnay et al., 2011). The second are bioinformatic tools, which are now extensively used in clinical diagnosis. However, the computational methods mainly based on evolutionary conservation and protein structure, have limitations (Dorfman et al., 2010). Notably, for the prediction of splicing defects, the use of a combination of tools is more powerful than individual ones (Aissat et al., 2012, Raynal et al., 2013). In silico predictions need to be validated by either in vivo measures (when possible) or in vitro assays. Many in vitro assays are available today, exploring the effect of mutations on specific cellular processes. Indeed, splicing defects are reproduced using minigenes, and defects in transcriptional level estimated by reporter gene assays. CFTR processing and stability are monitored by biochemical assays such as western blot and surface biotinylation. Protein localization can be visualized by immuno-cytochemistry and channel function measured by electrophysiological techniques (Ussing chamber, halide-sensitive assays or patch-clamp). While these tools enable to correctly identify major defects, subtle changes in the individual cellular processes tested are more difficult to interpret.

Indeed, it must be pointed out that $1-1.5 \%$ of $\mathrm{CF}$ alleles remain undetermined in patients with classic CF (Castellani et al., 2008). The classification and assignment of CFTR mutations to CF patients is the basis for current and future genotype-based therapeutic strategies.

\section{Current mutation-targeted therapeutic strategies}

As our understanding of the genetics of CF grows, and as technological advances render molecular diagnostic tools more powerful, many $\mathrm{CF}$ researchers worldwide now focus on the development of drugs that aim to treat CF by targeting the underlying cause of the disease. These drugs include molecules inducing readthrough of premature termination codons, potentiator molecules, as well as corrector molecules, presented hereafter.

\subsection{Molecules inducing readthrough of PTC}

Premature termination codons usually induce the destruction of the PTC-harbouring transcript through the NMD pathway; however, some PTCs can lead to the production of a truncated protein. In the nucleus, the removal of the introns leaves an exon junction complex (EJC) located 
at each exon-exon boundary. The removal of these complexes by the ribosome during the first round of translation serves to monitor the pre-mRNA. When a transcript harbours a PTC, the ribosome will stall at this particular position and leave downstream EJCs. The EJC will then recruit UPF1, via its interaction with other UPF proteins (UPF2 and UPF3/3X (or UPF3a or UPF3b)). This in turn will lead to the degradation of the PTC containing transcript by the NMD pathway (Amrani et al., 2006, Bidou et al., 2012, Maquat, 2004). This model predicts the recognition of PTCs located in all exons, with the exception of the last exon. Additionally, PTCs located in the last 50 nucleotides of the penultimate exon were shown to bypass NMD recognition, probably due to steric hindrances with the EJC. Hence, PTCs have been classified as either NMD-sensitive or NMD-irrelevant depending on their position. More recent data challenged this model, as some NMD-irrelevant transcripts were found to be degraded, whereas some NMD-sensitive transcripts were not. This led to the Faux-3' UTR model, where mRNA degradation by NMD is dependent on the distance between the PTC and the Poly(A)-binding proteins (PABPs) (Eberle et al., 2008, Silva et al., 2008). According to this model, the latter distance will influence the kinetics of dissociation of the ribosome/polypeptide complex, affecting the recruitment of UPF1 (Muhlemann, 2008). In any case, most transcripts harbouring PTCs will be degraded, preventing the production of deleterious truncated proteins.

Small molecules have been shown to induce readthrough of PTCs. Readthrough therapy targets a molecular defect common to all genetic diseases. It could therefore treat a substantial proportion of patients as in-frame nonsense mutations account for $12 \%$ of all hereditary diseasecausing mutations (Kellermayer, 2006). Studies have been performed in both in vitro models (over 45 different genes in more than 80 studies) and in clinical trials (8 different genes in 16 studies) (reviewed in (Lee and Dougherty, 2012)).

Compounds inducing readthrough favor an improper recognition of the PTC and the recruitment of a near cognate tRNA in the A site of the ribosome in place of the termination complex. If enough PTCs are recoded into sense-codons, enough full-length, functional protein may then be restored to provide a therapeutic benefit.

The best characterized drugs active against PTCs are aminoglycosides. Studies demonstrated the restoration of CFTR-dependent $\mathrm{Cl}^{-}$secretion and protein expression with improved clinical endpoints (Sermet-Gaudelus et al., 2007, Wilschanski et al., 2003). PTC124 (trade name Ataluren) was more recently identified in a high throughput screen (Welch et al., 2007). This 
molecule was found to suppress nonsense mutations more efficiently than aminoglycosides without nephro- or oto-toxic side effects. A double-blind, placebo-controlled Phase III study is now under evaluation. Novel readthrough molecules are being identified, such as RTC13 and RTC14 (Du et al., 2009) or NB124 (Rowe et al., 2011). Interestingly, an NMD inhibitor, amlexanox, has recently been shown to induce readthrough (Gonzalez et al., 2012). Such drugs presenting dual activities could increase readthrough efficiency by increasing the amount of target transcripts.

Before these molecules can be used in routine therapy, certain aspects of their mechanisms of action still need to be understood. Among these, what will be their consequence on normal stop codons? It appears that the rapid dissociation of the terminating ribosome at the normal stop codon does not enable efficient readthrough. This is comforted by the absence of elongated proteins after PTC124 treatment (Welch et al., 2007). Additionally, if the ribosome decodes the 3' UTR sequence, it will either rapidly encounter an in-frame stop codon or will decode the polyA-tail, leading to the synthesis of a poly-Lysine tail at the end of the nascent protein. This, in turn, initiates a quality control mechanism named non-stop decay, leading in fine to the degradation of both the polypeptide and the transcript (Graille and Seraphin, 2012).

Efficiency of readthrough therapy is linked to various parameters, some of which are predictable while others are measurable. Predictions can be made knowing the identity of the stop codon ( $\mathrm{UAA}<\mathrm{UAG}<\mathrm{UGA}$ ) and the local nucleotide sequence, where nucleotides in position -1 and +4 appear to have the greatest effect (Floquet et al., 2012). The amount of target transcripts, dependent on the level of NMD, also directly influences readthrough efficiency; hence a positive correlation has been obtained between $\mathrm{Cl}^{-}$transport modification and the level of increase in CFTR transcript after gentamicin nasal application (Linde et al., 2007). Skipping of the PTC harboring exon should also be taken into account as it will also reduce the amount of target transcripts (Hinzpeter et al., 2013). Indeed, taking into account these two parameters, it appeared that $8 \%$ of the CFTR transcripts would be targeted by readthrough therapy in a patient homozygous for the p.Glu831X mutation, which would be predictive of poor treatment efficiency (Hinzpeter et al., 2010).

Finally, readthrough of a PTC leads to the incorporation of a random amino acid at the considered position, leading to the production of a pool of full-length proteins bearing a maximum of one single amino acid substitutions. These substitutions will lead to either 
functional or non-functional proteins. Therefore, PTCs located in key regions implicated in protein processing or function would be less efficiently corrected, even if high levels of expression can be achieved.

\subsection{Potentiator therapies or activation of CFTR channels}

Molecules enhancing channel activity, such as molecules belonging to the phytoestrogen family (genistein and isoflavone), have been identified. A high-throughput screen enabled the identification of VX-770, Ivacaftor (trade name Kalydeco, Vertex Pharmaceuticals). This molecule specifically targets the p.Gly551Asp gating mutation by increasing the channel open probability and consequently the flow of ions transported through the channel (Ramsey et al., 2011), (Eckford et al., 2012). It also has the ability to increase the open probability of the wildtype channel and other mutants, namely p.Phe508del (Van Goor et al., 2009) and p.Arg117His (Van Goor et al., 2012). This molecule will thus also benefit patients retaining low level wildtype CFTR expression, e.g. class V mutations. Finally, it has recently been proposed that a reduction of CFTR activity could lead to a CF-like acquired disease. Cigarette smokers presenting with or without Chronic Obstructive Pulmonary Disease (COPD) showed an increased sweat chloride concentration and reduced intestinal CFTR currents (Raju et al., 2013). This can be explained by a reduced transcriptional expression of CFTR (Cantin et al., 2006) and a reduced stability of the protein (Clunes et al., 2012). As CFTR potentiators have been shown to increase wild-type CFTR activity, it would be tempting to increase CFTR activity under such conditions.

\subsection{Corrector therapies or routing CFTR to the plasma membrane}

Bringing misfolded p.Phe508del protein to the cell surface is a major goal for the treatment of $\mathrm{CF}$. Some chemical compounds have been reported to allow the protein to evade endoplasmic reticulum quality control, enabling surface expression. These molecules named correctors, have been identified by high-throughput screening, and include molecules such as benzo[c]quinolizinium (MPB) compounds, Corr4a or VX-809 (Grove et al., 2009, Pedemonte et al., 2005, Pedemonte et al., 2011, Ye et al., 2010). The most promising, VX-809 can restore up to $15 \%$ of non-CF channel activity in primary respiratory epithelia (Van Goor et al., 2011, Van Goor et al., 2006). Recently, VX-809 has been shown to correct processing mutations located within the N-terminus of the protein more efficiently. Indeed, some of these processing mutants could be fully corrected under the tested conditions as compared to a maximum $15 \%$ correction 
obtained for p.Phe508del (Ren et al., 2013). Certain correctors could therefore be best suited for specific genotypes.

It must be pointed out that all these correctors have a ceiling efficacy in restoring chloride conductance of approximately 10-15 \% (Pedemonte et al., 2005, Van Goor et al., 2006).

Efficiency could be increased by either combining molecules, by targeting distinct folding steps or by identifying new more potent correctors. Interestingly, some correctors have been shown to be active on other misfolded ABC proteins, namely on the p.Gln141Lys gout-causing mutation in the $A B C G 2$ gene (Woodward et al., 2013). This knowledge opens the application of these drugs to other genetic syndromes.

\subsection{Combinatory therapies to optimize efficiency}

As mutations may induce several CFTR dysfunctions, distinct drugs could correct individual defects. For example, p.Phe508del CFTR, if located at the plasma membrane, also presents a gating defect (Dalemans et al., 1991). Therefore, potentiators aimed at normalizing defective p.Phe508del CFTR $\mathrm{Cl}^{-}$channel gating may increase the effect of correctors, which rescue the traffic of the protein to the membrane. More generally, potentiators would be beneficial for many classes of mutation. Indeed, they could activate channels produced after suppression therapies for class I mutations or residual wild-type channels expressed in class V mutations. Splicing modulators, such as antisense oligonucleotides, could increase the amount of these latter channels. Combinatory therapies may also be considered for compound heterozygotes.

\section{Conclusion}

$\mathrm{CF}$ is a complex disease with a broader clinical and genetic spectrum than previously thought. This is making CF diagnosis (classic vs. non-classic CF), carrier screening and prenatal screening decisions more and more difficult.

New technologies and research approaches currently enable CF researchers to work at developing drugs that target the dysfunction of the CFTR protein directly. Our knowledge of the existing CFTR mutations and their consequences, both at the structure-function level (how and why the CFTR protein is non-functional) and at the clinical level (what organ(s) are most affected by the mutation), will be essential in order to perfect the design of the new molecules that have mainly been identified by high-throughput screening to date. This information is needed not only for the development of the molecules themselves, but also for the optimization of their delivery to the patient (pill vs. nebulizer etc.). 
With our current knowledge, it is still very difficult to associate a specific CFTR mutation to a specific dysregulation of the structure and/or function of the CFTR protein. It is very unlikely that most CF patients may be treated with a single drug. As advances in CF research and development will provide solutions to these challenges, the future gold standard for CF treatment strategy will be a customized combinatory one, targeting both the clinical symptoms and the underlying genetic cause for each $\mathrm{CF}$ patient.

\section{Acknowledgments}

The authors thank Dr. Fabrice Lejeune for the critical reading of this manuscript and helpful discussions. The authors thank the French association Vaincre la Mucoviscidose for its continuous support.

\section{References}

Abou Tayoun AN, Tunkey CD, Pugh TJ, Ross T, Shah M, Lee CC, et al. A comprehensive assay for CFTR mutational analysis using next-generation sequencing. Clin Chem. 2013;59:1481-8.

Aissat A, de Becdelievre A, Golmard L, Vasseur C, Costa C, Chaoui A, et al. Combined computational-experimental analyses of CFTR exon strength uncover predictability of exon skipping level. Hum Mutat. 2012;revised.

Amrani N, Dong S, He F, Ganesan R, Ghosh S, Kervestin S, et al. Aberrant termination triggers nonsense-mediated mRNA decay. Biochem Soc Trans. 2006;34:39-42.

Andersen DH. Cystic fibrosis of the pancreas and its relation to celiac disease. Am J Dis Child. 1938;56:344-99.

Anderson MP, Welsh MJ. Regulation by ATP and ADP of CFTR chloride channels that contain mutant nucleotide-binding domains. Science. 1992;257:1701-4.

Bidou L, Allamand V, Rousset JP, Namy O. Sense from nonsense: therapies for premature stop codon diseases. Trends Mol Med. 2012;18:679-88.

Boyle MP. Nonclassic cystic fibrosis and CFTR-related diseases. Curr Opin Pulm Med. 2003;9:498-503.

Cantin AM, Hanrahan JW, Bilodeau G, Ellis L, Dupuis A, Liao J, et al. Cystic fibrosis transmembrane conductance regulator function is suppressed in cigarette smokers. Am J Respir Crit Care Med. 2006;173:1139-44.

Castellani C, Cuppens H, Macek M, Jr., Cassiman JJ, Kerem E, Durie P, et al. Consensus on the use and interpretation of cystic fibrosis mutation analysis in clinical practice. J Cyst Fibros. 2008;7:179-96.

Choi JY, Muallem D, Kiselyov K, Lee MG, Thomas PJ, Muallem S. Aberrant CFTR-dependent HCO3- transport in mutations associated with cystic fibrosis. Nature. 2001;410:94-7. 
Chu CS, Trapnell BC, Curristin S, Cutting GR, Crystal RG. Genetic basis of variable exon 9 skipping in cystic fibrosis transmembrane conductance regulator mRNA. Nat Genet. 1993;3:151-6.

Clain J, Fritsch J, Lehmann-Che J, Bali M, Arous N, Goossens M, et al. Two mild cystic fibrosisassociated mutations result in severe cystic fibrosis when combined in cis and reveal a residue important for cystic fibrosis transmembrane conductance regulator processing and function. $\mathrm{J}$ Biol Chem. 2001;276:9045-9.

Clain J, Lehmann-Che J, Dugueperoux I, Arous N, Girodon E, Legendre M, et al. Misprocessing of the CFTR protein leads to mild cystic fibrosis phenotype. Hum Mutat. 2005a;25:360-71.

Clain J, Lehmann-Che J, Girodon E, Lipecka J, Edelman A, Goossens M, et al. A neutral variant involved in a complex CFTR allele contributes to a severe cystic fibrosis phenotype. Hum Genet. 2005b;116:454-60.

Claustres M, Altieri JP, Guittard C, Templin C, Chevalier-Porst F, Des Georges M. Are p.I148T, p.R74W and p.D1270N cystic fibrosis causing mutations? BMC Med Genet. 2004;5:19.

Clunes LA, Davies CM, Coakley RD, Aleksandrov AA, Henderson AG, Zeman KL, et al. Cigarette smoke exposure induces CFTR internalization and insolubility, leading to airway surface liquid dehydration. Faseb J. 2012;26:533-45.

Cui L, Aleksandrov L, Chang XB, Hou YX, He L, Hegedus T, et al. Domain interdependence in the biosynthetic assembly of CFTR. J Mol Biol. 2007;365:981-94.

Dalemans W, Barbry P, Champigny G, Jallat S, Dott K, Dreyer D, et al. Altered chloride ion channel kinetics associated with the delta F508 cystic fibrosis mutation. Nature. 1991;354:526-8.

De Boeck K, Wilschanski M, Castellani C, Taylor C, Cuppens H, Dodge J, et al. Cystic fibrosis: terminology and diagnostic algorithms. Thorax. 2006;61:627-35.

Divac A, Nikolic A, Mitic-Milikic M, Nagorni-Obradovic L, Petrovic-Stanojevic N, DopudjaPantic V, et al. High frequency of the R75Q CFTR variation in patients with chronic obstructive pulmonary disease. J Cyst Fibros. 2004;3:189-91.

Dorfman R, Nalpathamkalam T, Taylor C, Gonska T, Keenan K, Yuan XW, et al. Do common in silico tools predict the clinical consequences of amino-acid substitutions in the CFTR gene? Clin Genet. 2010;77:464-73.

Du L, Damoiseaux R, Nahas S, Gao K, Hu H, Pollard JM, et al. Nonaminoglycoside compounds induce readthrough of nonsense mutations. J Exp Med. 2009;206:2285-97.

Dugueperoux I, Bellis G, Lesure JF, Renouil M, Flodrops H, De Braekeleer M. Cystic fibrosis at the Reunion Island (France): spectrum of mutations and genotype-phenotype for the Y122X mutation. J Cyst Fibros. 2004;3:185-8.

Eberle AB, Stalder L, Mathys H, Orozco RZ, Muhlemann O. Posttranscriptional gene regulation by spatial rearrangement of the 3' untranslated region. PLoS Biol. 2008;6:e92.

Eckford PD, Li C, Ramjeesingh M, Bear CE. Cystic fibrosis transmembrane conductance regulator (CFTR) potentiator VX-770 (ivacaftor) opens the defective channel gate of mutant CFTR in a phosphorylation-dependent but ATP-independent manner. J Biol Chem. 2012;287:36639-49.

Farrell PM, Rosenstein BJ, White TB, Accurso FJ, Castellani C, Cutting GR, et al. Guidelines for diagnosis of cystic fibrosis in newborns through older adults: Cystic Fibrosis Foundation consensus report. J Pediatr. 2008;153:S4-S14.

Ferec C, Cutting GR. Assessing the Disease-Liability of Mutations in CFTR. Cold Spring Harb Perspect Med. 2012;2. 
Floquet C, Hatin I, Rousset JP, Bidou L. Statistical analysis of readthrough levels for nonsense mutations in mammalian cells reveals a major determinant of response to gentamicin. PLoS Genet. 2012;8:e1002608.

Gonzalez S, Beghyn T, Jia J, Debreuck N, Berte G, Mamchaoui K, et al. Rescue of nonsense mutations by amlexanox in human cells. Orphanet J Rare Dis. 2012;7:58.

Graille M, Seraphin B. Surveillance pathways rescuing eukaryotic ribosomes lost in translation. Nat Rev Mol Cell Biol. 2012;13:727-35.

Grove DE, Rosser MF, Ren HY, Naren AP, Cyr DM. Mechanisms for rescue of correctable folding defects in CFTRDelta F508. Mol Biol Cell. 2009;20:4059-69.

Haardt M, Benharouga M, Lechardeur D, Kartner N, Lukacs GL. C-terminal truncations destabilize the cystic fibrosis transmembrane conductance regulator without impairing its biogenesis. A novel class of mutation. J Biol Chem. 1999;274:21873-7.

He L, Aleksandrov LA, Cui L, Jensen TJ, Nesbitt KL, Riordan JR. Restoration of domain folding and interdomain assembly by second-site suppressors of the DeltaF508 mutation in CFTR. Faseb J. 2010;24:3103-12.

Hinzpeter A, Aissat A, de Becdelievre A, Bieth E, Sondo E, Martin N, et al. Alternative splicing of in-frame exon associated with premature termination codons: implications for readthrough therapies. Hum Mutat. 2013;34:287-91.

Hinzpeter A, Aissat A, Sondo E, Costa C, Arous N, Gameiro C, et al. Alternative splicing at a NAGNAG acceptor site as a novel phenotype modifier. PLoS Genet. 2010;6.

Jensen TJ, Loo MA, Pind S, Williams DB, Goldberg AL, Riordan JR. Multiple proteolytic systems, including the proteasome, contribute to CFTR processing. Cell. 1995;83:129-35.

Kellermayer R. Translational readthrough induction of pathogenic nonsense mutations. Eur J Med Genet. 2006;49:445-50.

Kerem B, Rommens JM, Buchanan JA, Markiewicz D, Cox TK, Chakravarti A, et al. Identification of the cystic fibrosis gene: genetic analysis. Science. 1989;245:1073-80.

Lee HL, Dougherty JP. Pharmaceutical therapies to recode nonsense mutations in inherited diseases. Pharmacol Ther. 2012;136:227-66.

Linde L, Boelz S, Nissim-Rafinia M, Oren YS, Wilschanski M, Yaacov Y, et al. Nonsensemediated mRNA decay affects nonsense transcript levels and governs response of cystic fibrosis patients to gentamicin. J Clin Invest. 2007;117:683-92.

Lucotte G, Hazout S, De Braekeleer M. Complete map of cystic fibrosis mutation DF508 frequencies in Western Europe and correlation between mutation frequencies and incidence of disease. Hum Biol. 1995;67:797-803.

Maquat LE. When cells stop making sense: effects of nonsense codons on RNA metabolism in vertebrate cells. RNA. 1995;1:453-65.

Maquat LE. Nonsense-mediated mRNA decay: splicing, translation and mRNP dynamics. Nat Rev Mol Cell Biol. 2004;5:89-99.

Mendoza JL, Schmidt A, Li Q, Nuvaga E, Barrett T, Bridges RJ, et al. Requirements for efficient correction of DeltaF508 CFTR revealed by analyses of evolved sequences. Cell. 2012;148:164-74.

Muhlemann O. Recognition of nonsense mRNA: towards a unified model. Biochem Soc Trans. 2008;36:497-501.

Pagani F, Baralle FE. Genomic variants in exons and introns: identifying the splicing spoilers. Nat Rev Genet. 2004;5:389-96. 
Pedemonte N, Lukacs GL, Du K, Caci E, Zegarra-Moran O, Galietta LJ, et al. Small-molecule correctors of defective DeltaF508-CFTR cellular processing identified by high-throughput screening. J Clin Invest. 2005; 115:2564-71.

Pedemonte N, Tomati V, Sondo E, Caci E, Millo E, Armirotti A, et al. Dual activity of aminoarylthiazoles on the trafficking and gating defects of the cystic fibrosis transmembrane conductance regulator chloride channel caused by cystic fibrosis mutations. J Biol Chem. 2011;286:15215-26.

Protasevich I, Yang Z, Wang C, Atwell S, Zhao X, Emtage S, et al. Thermal unfolding studies show the disease causing F508del mutation in CFTR thermodynamically destabilizes nucleotide-binding domain 1. Protein Sci. 2010;19:1917-31.

Rabeh WM, Bossard F, Xu H, Okiyoneda T, Bagdany M, Mulvihill CM, et al. Correction of both NBD1 energetics and domain interface is required to restore DeltaF508 CFTR folding and function. Cell. 2012;148:150-63.

Raju SV, Jackson PL, Courville CA, McNicholas CM, Sloane PA, Sabbatini G, et al. Cigarette Smoke Induces Systemic Defects in Cystic Fibrosis Transmembrane Conductance Regulator (CFTR) Function. Am J Respir Crit Care Med. 2013.

Ramsey BW, Davies J, McElvaney NG, Tullis E, Bell SC, Drevinek P, et al. A CFTR potentiator in patients with cystic fibrosis and the G551D mutation. N Engl J Med. 2011;365:1663-72.

Raynal C, Baux D, Theze C, Bareil C, Taulan M, Roux AF, et al. A classification model relative to splicing for variants of unknown clinical significance: application to the CFTR gene. Hum Mutat. 2013;34:774-84.

Reddy MM, Quinton PM. Selective activation of cystic fibrosis transmembrane conductance regulator Cl- and HCO3- conductances. JOP. 2001;2:212-8.

Ren HY, Grove DE, De La Rosa O, Houck SA, Sopha P, Van Goor F, et al. VX-809 corrects folding defects in cystic fibrosis transmembrane conductance regulator protein through action on membrane-spanning domain 1. Mol Biol Cell. 2013;24:3016-24.

Rosenstein BJ. Nonclassic cystic fibrosis: a clinical conundrum. Pediatr Pulmonol. 2003;36:10-2.

Rowe SM, Sloane P, Tang LP, Backer K, Mazur M, Buckley-Lanier J, et al. Suppression of CFTR premature termination codons and rescue of CFTR protein and function by the synthetic aminoglycoside NB54. J Mol Med (Berl). 2011;89:1149-61.

Schneider A, Larusch J, Sun X, Aloe A, Lamb J, Hawes R, et al. Combined bicarbonate conductance-impairing variants in CFTR and SPINK1 variants are associated with chronic pancreatitis in patients without cystic fibrosis. Gastroenterology. 2011;140:162-71.

Seibert FS, Linsdell P, Loo TW, Hanrahan JW, Riordan JR, Clarke DM. Cytoplasmic loop three of cystic fibrosis transmembrane conductance regulator contributes to regulation of chloride channel activity. J Biol Chem. 1996;271:27493-9.

Sermet-Gaudelus I, Renouil M, Fajac A, Bidou L, Parbaille B, Pierrot S, et al. In vitro prediction of stop-codon suppression by intravenous gentamicin in patients with cystic fibrosis: a pilot study. BMC Med. 2007;5:5.

Sheppard DN, Rich DP, Ostedgaard LS, Gregory RJ, Smith AE, Welsh MJ. Mutations in CFTR associated with mild-disease-form Cl- channels with altered pore properties. Nature. 1993;362:160-4.

Shoshani T, Augarten A, Gazit E, Bashan N, Yahav Y, Rivlin Y, et al. Association of a nonsense mutation (W1282X), the most common mutation in the Ashkenazi Jewish cystic fibrosis patients in Israel, with presentation of severe disease. Am J Hum Genet. 1992;50:222-8. 
Silva AL, Ribeiro P, Inacio A, Liebhaber SA, Romao L. Proximity of the poly(A)-binding protein to a premature termination codon inhibits mammalian nonsense-mediated mRNA decay. RNA. 2008;14:563-76.

Sosnay PR, Castellani C, Corey M, Dorfman R, Zielenski J, Karchin R, et al. Evaluation of the disease liability of CFTR variants. Methods Mol Biol. 2011;742:355-72.

Thauvin-Robinet C, Munck A, Huet F, Genin E, Bellis G, Gautier E, et al. The very low penetrance of cystic fibrosis for the $\mathrm{R} 117 \mathrm{H}$ mutation: a reappraisal for genetic counselling and newborn screening. J Med Genet. 2009;46:752-8.

Trujillano D, Ramos MD, Gonzalez J, Tornador C, Sotillo F, Escaramis G, et al. Next generation diagnostics of cystic fibrosis and CFTR-related disorders by targeted multiplex high-coverage resequencing of CFTR. J Med Genet. 2013;50:455-62.

Van Goor F, Hadida S, Grootenhuis PD, Burton B, Cao D, Neuberger T, et al. Rescue of CF airway epithelial cell function in vitro by a CFTR potentiator, VX-770. Proc Natl Acad Sci U S A. 2009; 106:18825-30.

Van Goor F, Hadida S, Grootenhuis PD, Burton B, Stack JH, Straley KS, et al. Correction of the F508del-CFTR protein processing defect in vitro by the investigational drug VX-809. Proc Natl Acad Sci U S A. 2011;108:18843-8.

Van Goor F, Straley KS, Cao D, Gonzalez J, Hadida S, Hazlewood A, et al. Rescue of DeltaF508-CFTR trafficking and gating in human cystic fibrosis airway primary cultures by small molecules. Am J Physiol Lung Cell Mol Physiol. 2006;290:L1117-30.

Van Goor F, Yu HD, Burton B, Huang CF, Hoffman B. Ivacaftor potentiates multiple mutant CFTR forms. Pediatr Pulmonol. 2012;47:Abstract 31.

Ward CL, Omura S, Kopito RR. Degradation of CFTR by the ubiquitin-proteasome pathway. Cell. 1995;83:121-7.

Welch EM, Barton ER, Zhuo J, Tomizawa Y, Friesen WJ, Trifillis P, et al. PTC124 targets genetic disorders caused by nonsense mutations. Nature. 2007;447:87-91.

Welsh MJ, Ramsey BW, Accurso F, Cutting GR. Cystic fibrosis. 8th ed. New York: McGrawHill; 2001.

Welsh MJ, Smith AE. Molecular mechanisms of CFTR chloride channel dysfunction in cystic fibrosis. Cell. 1993;73:1251-4.

WHO. Classification of cystic fibrosis and related disorders. Report of a joint WHO/ICF(M)A/ECFTN meeting. 2000;1:5-8.

Will K, Dork T, Stuhrmann M, von der Hardt H, Ellemunter H, Tummler B, et al. Transcript analysis of CFTR nonsense mutations in lymphocytes and nasal epithelial cells from cystic fibrosis patients. Hum Mutat. 1995;5:210-20.

Wilschanski M, Yahav Y, Yaacov Y, Blau H, Bentur L, Rivlin J, et al. Gentamicin-induced correction of CFTR function in patients with cystic fibrosis and CFTR stop mutations. N Engl J Med. 2003;349:1433-41.

Woodward OM, Tukaye DN, Cui J, Greenwell P, Constantoulakis LM, Parker BS, et al. Goutcausing Q141K mutation in ABCG2 leads to instability of the nucleotide-binding domain and can be corrected with small molecules. Proc Natl Acad Sci U S A. 2013;110:5223-8.

Ye L, Knapp JM, Sangwung P, Fettinger JC, Verkman AS, Kurth MJ. Pyrazolylthiazole as DeltaF508-cystic fibrosis transmembrane conductance regulator correctors with improved hydrophilicity compared to bithiazoles. J Med Chem. 2010;53:3772-81. 
Table 1. Classification of CFTR mutations according to their clinical consequences. Examples of each of the four groups of mutations are given.

\begin{tabular}{|c|c|c|c|}
\hline Group A & Group B & Group C & Group D \\
\hline $\begin{array}{l}\text { Classic-CF } \\
\text { CF-causing mutations }\end{array}$ & $\begin{array}{l}\text { Non-Classic CF } \\
\text { CFTR-related disorder } \\
\text { associated mutations }\end{array}$ & $\begin{array}{l}\text { No Clinical } \\
\text { Consequence }\end{array}$ & $\begin{array}{l}\text { Unknown } \\
\text { Clinical } \\
\text { Relevance }\end{array}$ \\
\hline $\begin{array}{l}\text { All mutations in } \\
\text { Table } 2 \text { and } \\
711+3 \mathrm{~A}>\mathrm{G}^{*}, \mathrm{R} 117 \mathrm{H}- \\
\text { T5*, D1152H*, } \\
\text { L206W*, TG13-T5* }\end{array}$ & $\begin{array}{l}\text { TG13-T5*, R117H-T5*, } \\
\text { D1152H*, L206W*, } \\
\text { L997F, M952I, D565G*, } \\
\text { TG11-T5**, R117H-T7**, } \\
\text { D443Y-G576A-R668C, } \\
\text { R74W-D1270N, R75Q** }\end{array}$ & $\begin{array}{l}\text { TG11-T5**, R117H- } \\
\text { T7**, R75Q**, } \\
875+40 A / G, M 470 V, \\
\text { T854T, P1290P, } \\
\text { I807M, I521F, } \\
\text { R74W, F508C, } \\
\text { I506V, I148T }\end{array}$ & $\begin{array}{l}\text { All mutations } \\
\text { (mostly } \\
\text { missense) not } \\
\text { yet analyzed or } \\
\text { undergoing } \\
\text { functional } \\
\text { analysis }\end{array}$ \\
\hline
\end{tabular}

* Mutations that may belong either to Group A or to Group B.

** Mutations that may belong either to Group B or to Group C.

Table 2. ACMG recommended panel of 23 classic CF-causing mutations. These mutations include missense, stop, splicing and frameshift mutations.

\section{ACMG recommended panel of classic CF-causing mutations}

\begin{tabular}{|l|l|l|l|l|}
\hline G85E & I507del & R560T & $621+1 \mathrm{G}>\mathrm{T}$ & $2789+5 \mathrm{G}>\mathrm{A}$ \\
R117H & F508del & R1162X & $711+1 \mathrm{G}>\mathrm{T}$ & $3120+1 \mathrm{G}>\mathrm{A}$ \\
R334W & G542X & W1282X & $1717-1 \mathrm{G}>\mathrm{A}$ & $3659 \mathrm{delC}$ \\
R347P & G551D & N1303K & $1898+1 \mathrm{G}>\mathrm{A}$ & $3849+10 \mathrm{kbC}>\mathrm{T}$ \\
A455E & R553X & & $2184 \mathrm{del} A$ & \\
\hline
\end{tabular}

Additional or alternative mutations present at significant frequencies in an ethnic population served by a newborn screening program may be assessed. 


\section{Figure 1}

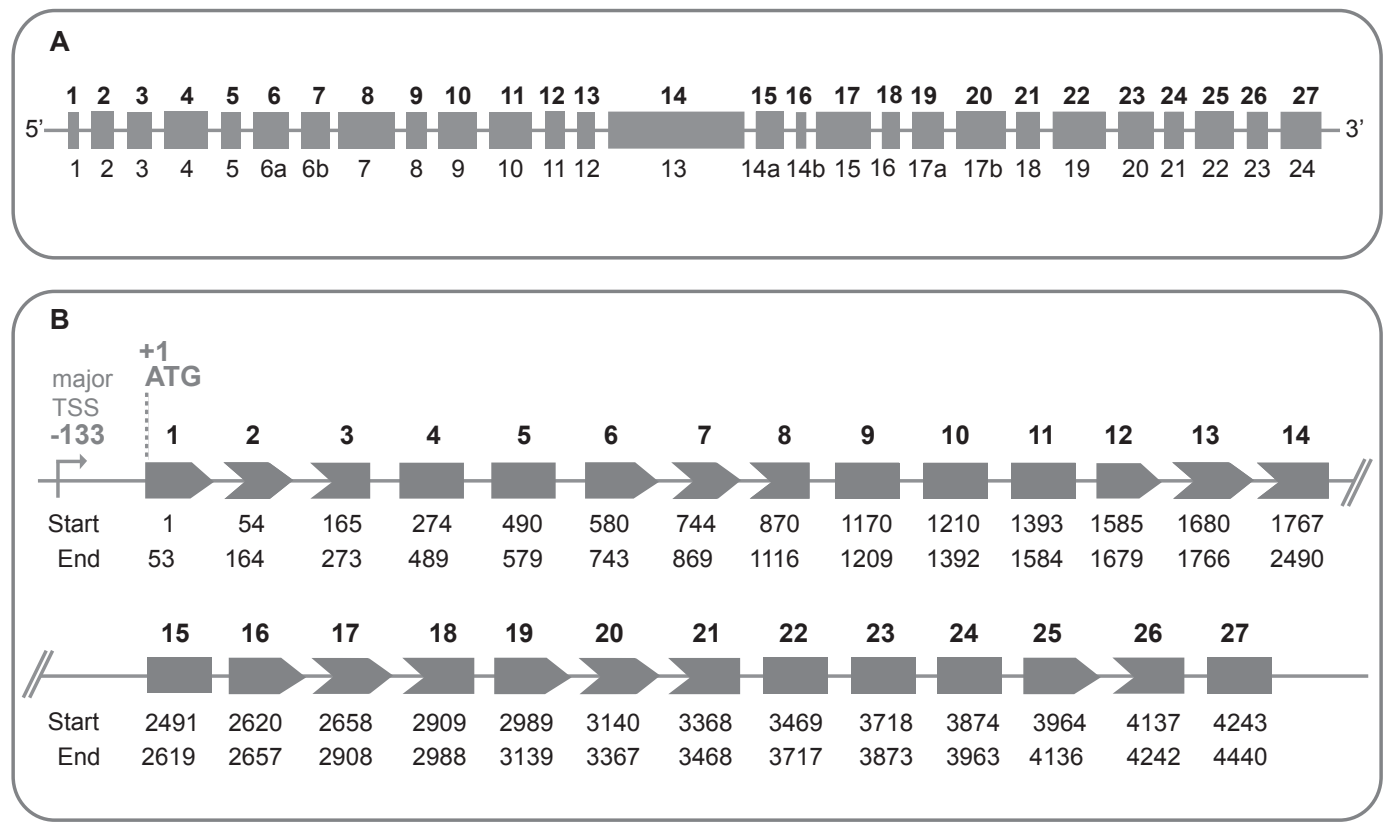

Fig. 1. Scheme of the CFTR gene. (A) Rescaled exon scheme with minimized introns, which are not drawn to scale. At the bottom in normal font, the exon labeling numbers, 1 through 24 , according to the old nomenclature. At the top in bold font, the exon labeling numbers, 1 through 27, according to the new nomenclature. (B) Correspondence numbering and reading frame for exons and nucleotides. In bold font, common numbering of exons, 1 through 27, according to the new nomenclature. First (start) and last (end) nucleotides are indicated for each exon. The major transcription start site (TSS) is indicated in grey at -133 base pairs from ATG $(+1)$. The flat edges indicate the exons with start and end nucleotides aligned with the reading frame; the arrow tips indicate exon starts and ends with frame shifted nucleotides. cDNA RefSeq NM_000492. 


\section{Figure 2}

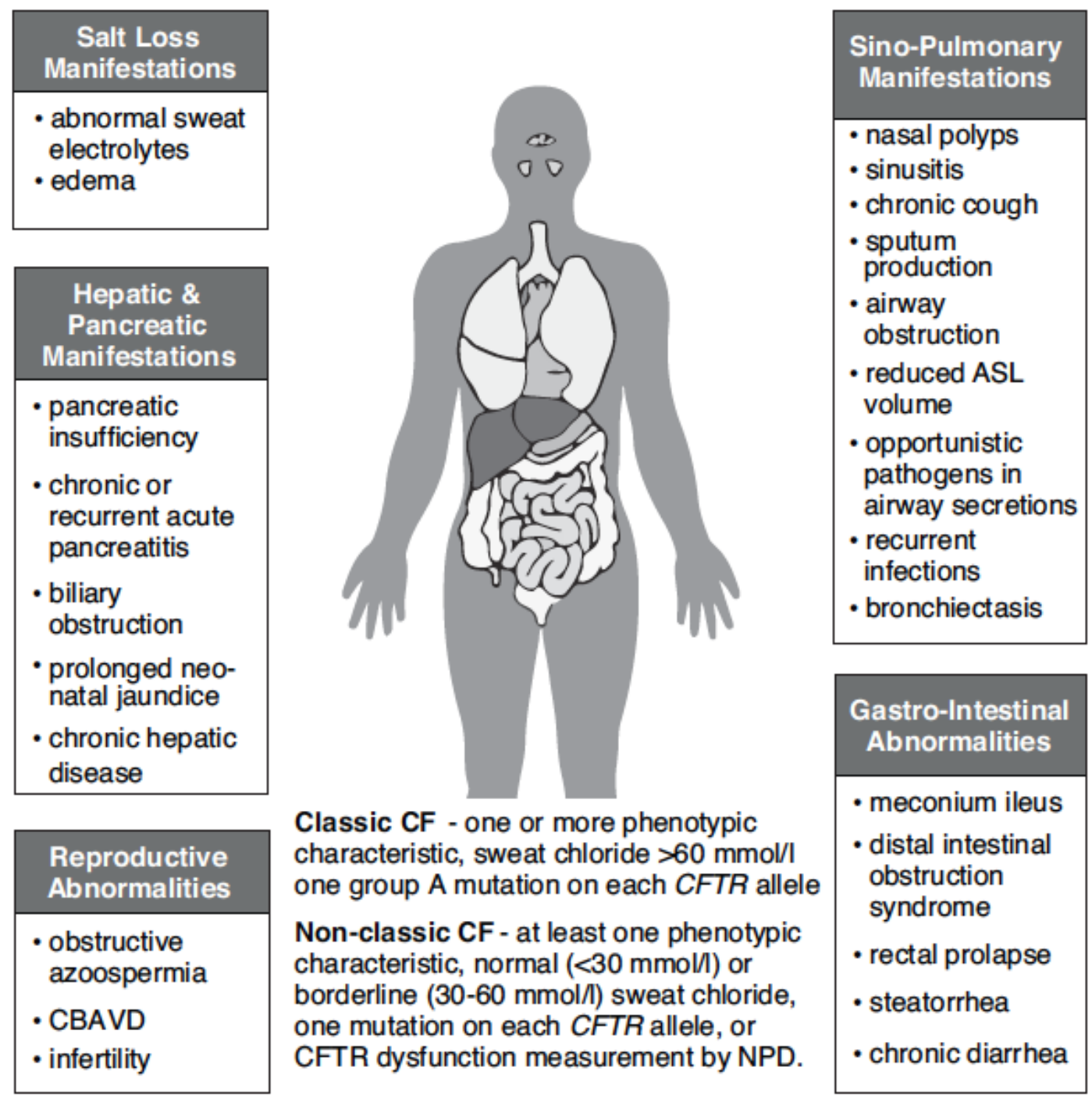

Fig. 2. The Clinical Spectrum of Cystic Fibrosis. This diagram depicts the phenotypic characteristics of CF and distinguishes between classic and non-classic CF clinical descriptions. 
Figure 3

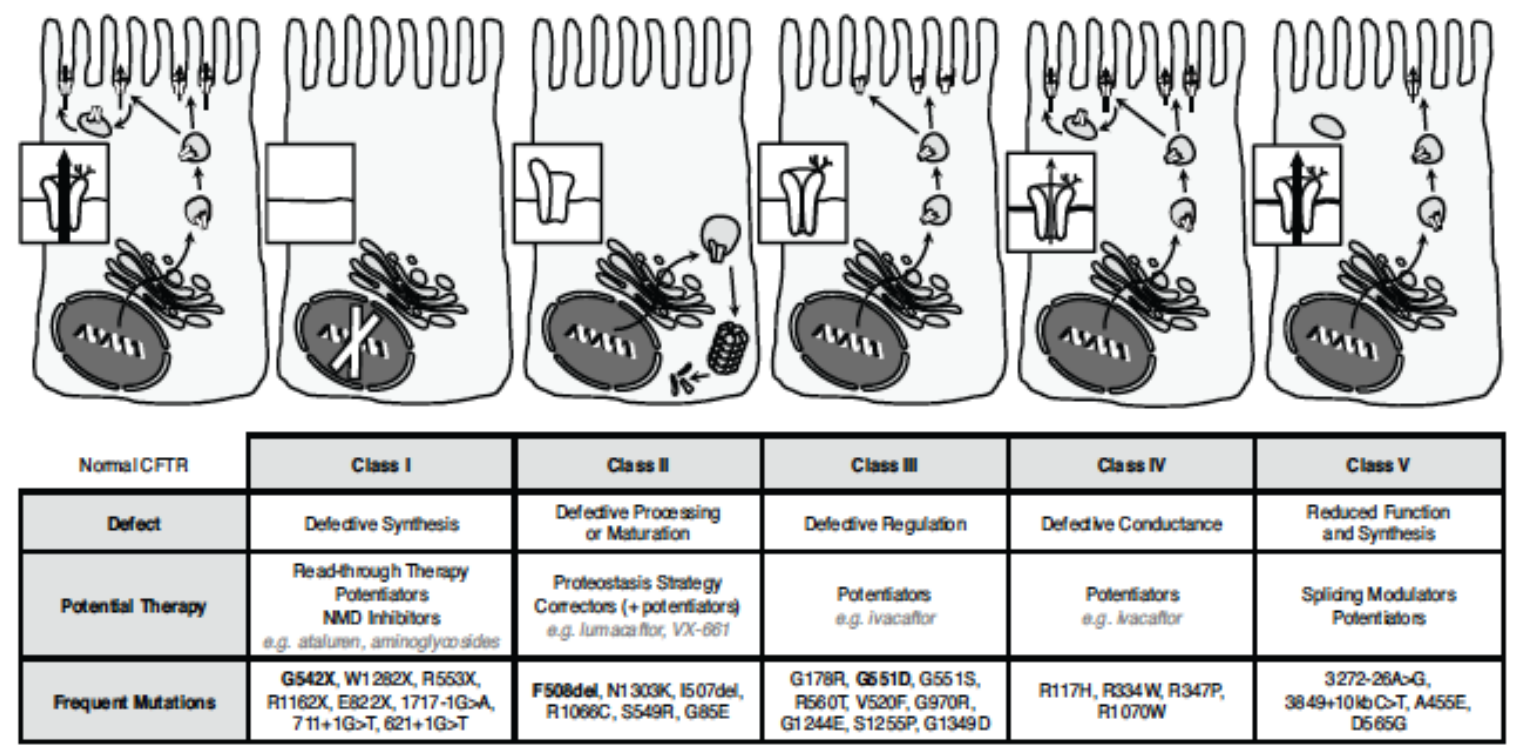

Fig.3. Classification of CF mutations according to CFTR structure and function assessment.

Each of the five classes of mutations are described. A cell illustration representing the

localization of mutated CFTR relative to normal / wild-type CFTR (cell at the very left) is shown for each mutation class, along with a description of the nature of the defect, potential therapies and frequent mutations for this particular class. In bold, the most prevalent mutation of each class. 